\title{
Microdeletion syndromes disclose replication timing alterations of genes unrelated to the missing DNA Josepha Yeshaya ${ }^{\dagger 1}$, Itay Amir ${ }^{\dagger 1,2}$, Ayelet Rimon ${ }^{1}$, Jane Freedman ${ }^{1}$, Mordechai Shohat ${ }^{1,2,3}$ and Lydia Avivi*2
}

Address: ${ }^{1}$ Raphael Recanati Genetic Institute, Rabin Medical Center Beilinson Campus, Petah-Tikva, Israel, ${ }^{2}$ Department of Human Molecular Genetics \& Biochemistry, Sackler School of Medicine, Tel-Aviv University, Tel-Aviv, Israel and ${ }^{3}$ Department of Pediatrics C, Schneider Children's Medical Center of Israel, Petah Tikva, Israel

Email: Josepha Yeshaya - JYeshaya@clalit.org.il; Itay Amir - itayamir@gmail.com; Ayelet Rimon - LabZitogen@clalit.org.il; Jane Freedman - LabZitogen@clalit.org.il; Mordechai Shohat - mshohat@post.tau.ac.il; Lydia Avivi* - lydia@post.tau.ac.il

* Corresponding author †Equal contributors

Published: 14 March 2009

Molecular Cytogenetics 2009, 2:1I doi:10.1186/1755-8166-2-II
Received: 2 January 2009

Accepted: I4 March 2009

This article is available from: http://www.molecularcytogenetics.org/content/2/I/I I

(C) 2009 Yeshaya et al; licensee BioMed Central Ltd.

This is an Open Access article distributed under the terms of the Creative Commons Attribution License (http://creativecommons.org/licenses/by/2.0), which permits unrestricted use, distribution, and reproduction in any medium, provided the original work is properly cited.

\begin{abstract}
Background: The temporal order of allelic replication is interrelated to the epigenomic profile. A significant epigenetic marker is the asynchronous replication of monoallelically-expressed genes versus the synchronous replication of biallelically-expressed genes. The present study sought to determine whether a microdeletion in the genome affects epigenetic profiles of genes unrelated to the missing segment. In order to test this hypothesis, we checked the replication patterns of two genes - SNRPN, a normally monoallelically expressed gene (assigned to I5qII.I3), and the RBI, an archetypic biallelically expressed gene (assigned to 13.q/4) in the genomes of patients carrying the $22 q$ I I.2 deletion (DiGeorge/Velocardiofacial syndrome) and those carrying the $7 q \mathrm{l} I .23$ deletion (Williams syndrome).
\end{abstract}

Results: The allelic replication timing was determined by fluorescence in situ hybridization (FISH) technology performed on peripheral blood cells. As expected, in the cells of normal subjects the frequency of cells showing asynchronous replication for SNRPN was significantly $\left(P<10^{-12}\right)$ higher than the corresponding value for $R B I$. In contrast, cells of the deletion-carrying patients exhibited a reversal in this replication pattern: there was a significantly lower frequency of cells engaging in asynchronous replication for SNRPN than for $R B I\left(\mathrm{P}<10^{-4}\right.$ and $\mathrm{P}<10^{-3}$ for DiGeorge/ Velocardiofacial and Williams syndromes, respectively). Accordingly, the significantly lower frequency of cells showing asynchronous replication for SNRPN than for RBI is a new epigenetic marker distinguishing these deletion syndrome genotypes from normal ones.

Conclusion: In cell samples of each deletion-carrying individual, an aberrant, reversed pattern of replication is delineated, namely, where a monoallelic gene replicates more synchronously than a biallelic gene. This inverted pattern, which appears to be non-deletion-specific, clearly distinguishes cells of deletion-carriers from normal ones. As such, it offers a potential epigenetic marker for suspecting a hidden microdeletion that is too small to be detected by conventional karyotyping methods. 


\section{Background}

Microdeletion syndromes are defined as viable human (constitutional) chromosomal aberrations, each resulting from a small hemizigous loss of DNA (ranging 1-5 Mb). These losses cannot be detected by conventional karyotyping methods, whose best resolution is no greater than 5 $\mathrm{Mb}[1,2]$. The various microdeletion syndromes are estimated to be among the major causes of mental retardation following Down and Fragile $\times$ syndromes [3]. Each is associated with multiple abnormalities in various organ systems, none of which, except for the missing DNA material, is specific or unique to the microdeletion in question, as all occur in the general population as well. However, what makes each syndrome specific and unique is the sum total of the abnormalities observed in each condition, although in the total population of patients each syndrome abnormality is highly variable in its presentation. This is exemplified by the wide spectrum of phenotypic variations characterizing DiGeorge/Velocardiofacial syndrome (DGS/VCFS), and by the variability in expression of Williams syndrome, each of which results from a submicroscopic loss of a tiny chromosomal segment. The large spectrum of anomalies in DGS/VCFS is associated with a deletion in chromosome band 22q11.2, while the variable clinical expression in Williams syndrome results from a deletion in 7q11.23 [4-7]. This permits to put forward the hypothesis that, in addition to the direct impact of the missing DNA, the "tiny monosomic status" by itself may also be implicated in the prenatal malformations and postnatal defects that define the affected phenotype. In view of that, a quantitative imbalance per se resulting from the missing DNA may disrupt the normal behavior of other DNA sequences that are present in the aberrant genome in their normal two doses. If, indeed, the loss of one copy of a tiny chromosomal segment can cause a broad spectrum of genomic malfunctions, one should expect to find various non-deletion-specific alterations in the epigenetic properties of various genes (including those situated on chromosomes other than that on which the deletion is situated). To test this hypothesis we examined the temporal order of allelic replication, a marker reflecting epigenetic qualities [reviewed in [8]; see also the next paragraph], of two unrelated genes, SNRPN on chromosome 15q11-13 and RB1 on chromosome 13q14, in two microdeletion syndromes, DGS/VCFS and Williams syndrome. These two syndromes were chosen because they differ in the missing DNA information, as well as the chromosome on which the missing DNA is located.

A dominant aspect of the inherent epigenomic profile is the exact timing of DNA synthesis at the S-phase of the cell cycle [9-11]. Usually, active loci replicate earlier than silent ones $[9,12]$. An archetypal example of the close correlation between replication timing and epigenetic inactivity is the difference in replication timing between the active and inactive X-chromosomes in mammalian female cells, whereby the inactive chromosome replicates considerably later than its active counterpart $[13,14]$. Furthermore, the close connection between replication timing and epigenetic silencing is apparent from the fact that the allelic counterparts of monoallelically expressed genes replicate asynchronously, while the allelic counterparts of biallelically expressed genes replicate synchronously $[8,15,16]$. A simple method for evaluating the temporal order of allelic replication is the fluorescent in situ hybridization (FISH) assay $[17,18]$. With this assay, it has been demonstrated that allelic counterparts of monoallelically expressed genes show asynchronous replication, with the active allele replicating earlier than the inactive counterpart [18-22]. The asynchronous pattern of allelic replication has been demonstrated in all known types of monoallelically expressed genes. These include: (i) imprinted genes, well exemplified by the SNRPN gene located within the Prader-Willi/Angelman syndrome imprinted region, which shows early replication of the active (paternal) allele and late replication of the inactive (maternal) allele [18-22]; (ii) genes subjected to X-chromosome inactivation [23-25]; and (iii) genes undergoing allelic exclusion $[8,26-34]$. Therefore, the timing of replication of two allelic counterparts (either synchronous or asynchronous), not necessarily in the tissue of expression, is considered to be a marker for the epigenetic properties of the gene in question [30,32-34].

In this study, we have shown in genomes containing a microdeletion, that certain genes, even those unrelated to the deletion, change their characteristic replication properties. Specifically, an imprinted gene (SNRPN) reveals relaxation of the asynchronous pattern of replication, characterizing monoallelic expression, whereas genes (RB1 and ARSA) that normally show synchronous patterns of replication alter their inherent mode and replicate asynchronously. We also found that when a gene replicates asynchronously due to the presence of a deletion, the preference of an allele for early or delayed replication is random.

\section{Methods \\ Subjects and cell cultures}

Peripheral blood samples obtained from 58 individuals belonging to three different groups of subjects who were referred for cytogenetic testing were used in this study. The first group comprised 30 healthy individuals taken among couples referring to the cytogenetic laboratory due to recurrent pregnancy loss, all of whom were found to have a normal karyotype (designated $\mathrm{C} 1-\mathrm{C} 30$ ); the second group consisted of 18 patients diagnosed with DGS/VCFS (designated V1-V18), all of whom carried the definitive 22q11.2 deletion confirmed by FISH analysis (Figure 1); and the third group included ten patients with Williams 


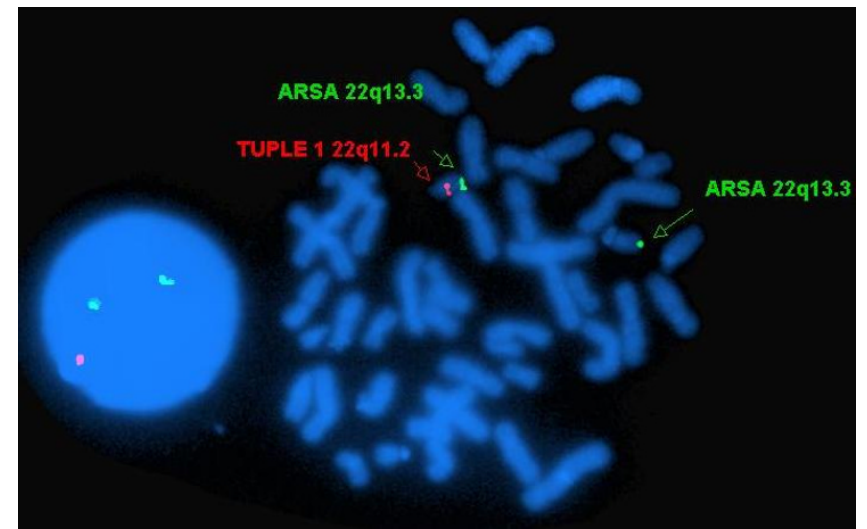

Figure I

Cells from a patient with DGS/VCFS, following twocolor FISH with TUPLEI (red) and ARSA (green).

Right, a metaphase-spread with the normal chromosome 22 , identified by double labeling (red and green signals) and the deletion-carrying one, distinguished by the absence of the red signal; left, an interphase cell exemplifying two ARSA (green) signals and a single TUPLEI (red) signal. The green signal that is closer to the red was assumed to belong to the normal homologue, while the more distant one to the deletion-carrying homologue.

syndrome (cases W1-W10), each of whom carried the characteristic 7q11.23 deletion confirmed by FISH. All the samples were incubated for 72 hours in RPMI 1640 medium supplemented with $20 \%$ fetal calf serum (FCS), $3 \%$ phytohemagglutinin (PHA) and 1\% antibiotics (a standard solution of penicillin, streptomycin and nystatin) at $37^{\circ} \mathrm{C}$. Eight control samples (C1-C8), one sample from the DGS/VCFS group (V1) and one sample from the Williams syndrome group (W1) were divided into two cultures, and one culture of each was pulsed-labeled at the S-phase by adding 5'-bromo-2'-deoxyuridine (BrdU; MP Biomedicals, Irvine, CA). At the end of the incubation period, BrdU was added to these cultures at a final concentration of $10^{-5} \mathrm{M}$ for $90 \mathrm{~min}$ at $37^{\circ} \mathrm{C}$, followed by a wash with phosphate buffered saline (PBS). Colcemid (Biological Industries, K. Beit-Haemek, Israel) was then added to all the samples, both labeled and unlabeled with BrdU, at a final concentration of $0.1 \mu \mathrm{g} / \mathrm{ml}$ for $20 \mathrm{~min}$, followed by hypotonic treatment $\left(0.075-\mathrm{M} \mathrm{KCL}\right.$ at $37^{\circ} \mathrm{C}$ for $\left.15 \mathrm{~min}\right)$ and five washes, each with a fresh cold 3:1 methanol:acetic acid solution.

\section{Probes}

Three directly labeled chromosomal probes from Vysis (Doweners Grove, IL, USA) were used: (i) the RB1 probe (32-192018) located on chromosome 13q14; (ii) the SNRPN probe (32-190004) located on chromosome 15q11-13; and (iii) the green-labeled ARSA probe (32191028 ) located on chromosome 22q13.3, which is com- mercially mixed with the red-labeled TUPLE1 probe located on chromosome 22q11.2. In cases with an absence of one copy of 22q11.2, this two-probe combination enabled us, even at interphase, to determine whether an ARSA signal was located on the homologue containing the deletion or on the normal partner (Figure 1).

\section{In-situ hybridization}

Each probe was mixed with a hybridization solution and poured onto the fresh slide spreads, covered with a $12 \mathrm{~mm}$ cover slip and sealed with rubber cement. Co-denaturation was performed at $76^{\circ} \mathrm{C}$ for six min followed by $18 \mathrm{hrs}$ hybridization at $37^{\circ} \mathrm{C}$ in a moist chamber. Following hybridization the slides were washed in $0.4 \times \mathrm{SSC} / 0.3 \%$ $\mathrm{NP} 40$ at $53^{\circ} \mathrm{C}$ for $2 \mathrm{~min}$ and in $2 \times \mathrm{SSC} / 0.1 \% \mathrm{NP} 40$ at room temperature for $1 \mathrm{~min}$. After the second washing the slides with the BrdU-labeled samples were incubated with a blocking solution consisting of $1 \%$ Bovine serum albumin (BSA, Boerhinger-Mannheim) in $4 \times \mathrm{SSC}$ at $37^{\circ} \mathrm{C}$ for $30 \mathrm{~min}$. At the same time the non-labeled slides were counterstained with DAPI (4,6-diamidino-2-phenylindole, Vector Laboratories Inc., Burlingame, CA) antifade solution and analyzed for simultaneous viewing of FITC, Texas red and DAPI.

\section{Detection of BrdU-labeled samples}

After blocking, BrdU was detected by anti mouse antibody conjugated to AMCA (Jackson Immunoresearch Laboratories, West Grove, PA, USA) linked by mouse anti-BrdU antibody (Dako Cytomation, Glostrup, Denmark). The two antibodies were diluted to the appropriate concentrations in a solution of $4 \times$ SSC with $1 \%$ BSA. The slides were incubated with each antibody for $30 \mathrm{~min}$ at $37^{\circ} \mathrm{C}$, and each incubation was followed by 3 washes in PBS/ $0.1 \%$ Triton $\times 100$ at room temperature for $5 \mathrm{~min}$. After the final washing, the slides were mounted with an antifade solution (Vectashield, Vector Laboratories, Burlingame, CA

\section{Cytogenetic evaluation}

The slides were analyzed blindly on an Olympus BX51 fluorescent microscope fitted with a triple band-pass filter (Chroma Technology, Brattleboro, VT, USA) for the simultaneous detection of three colors: blue-DAPI and AMCA, green-FITC and red-rhodamine or Texas Red.

We used the FISH replication assay to determine whether the replication patterns of three loci, RB1, SNRPN and $A R S A$, in samples of control individuals and patients with microdeletion syndromes were synchronous or asynchronous. For each case, each probe and each BrdU-labeled and unlabeled sample we analyzed at least 100 interphase cells that showed, following FISH, two clearly stained hybridization signals (Figure 2). We noted the structure of each fluorescent signal in these cells - i.e. whether it was 
a singlet (S), representing a non-replicated sequence, or a doublet (D), indicating a replicated sequence. Accordingly, the cells were classified into three categories: (i) cells with two singlets (SS cells; Figure 2a, b, c); cells with two doublets (DD cells; Figure 2d, e, f); and (iii) cells with one singlet and one doublet (SD cells; Figure $2 \mathrm{~g}, \mathrm{~h}, \mathrm{i}$ ). Thus, in a population of replicating cells, following hybridization with a locus specific probe, the frequency (\%) of cells containing two signals differing in their replication status (SD cells), out of the total population of cells with two hybridization signals, represents the level of asynchrony in the replication timing of the allelic counterparts of the locus in question.

In order to differentiate in the interphase cell samples of the DGS/VCFS patients between the ARSA locus located on the deletion-carrying homologue and the one situated on the normal chromosome, we used the signal of the TUPLE1 allele as a reference point. We assumed that the ARSA allele that is closer to the TUPLE1 allele is located on the normal chromosome whereas its counterpart is located on the chromosome carrying the deletion (Figure $1)$.

\section{Statistical method}

The statistical significance of the differences between the two cell populations tested was determined by the twotailed Student's $t$-test.

\section{Ethical basis}

The study was approved by the Rabin Medical Center Review Board

\section{Results}

The frequency of SD cells for the RB1 locus in PHA-stimulated lymphocytes of control subjects (cases C1-C15) ranged from $16 \%$ to $30 \%$ with a mean and standard deviation value of $20.3 \pm 3.7 \%$ (Figure $3 a$ ). Specifically, in 14 out of the 15 control subjects tested the RB1 locus showed a low frequency of SD cells (less than 25\%) (Figure 3a). In contrast, as expected for an imprinted locus, in a group of 10 control subjects (cases C16-C25) the frequency of SD cells for the SNRPN locus was almost twice as high as that found for the RB1 locus, ranging from $44 \%$ to $57 \%$ with a mean and standard deviation value of $50.3 \pm 4.2 \%$ (Figure $3 \mathrm{~b})$. Clearly, in cells of control subjects the imprinted SNRPN locus shows significantly higher frequencies of SD cells compared to RB1 $\left(\mathrm{P}<10^{-12}\right)$.

However, in the cell samples from the DGS/VCFS and the Williams syndrome patients, the frequency of SD cells for the imprinted SNRPN locus were significantly lower than the corresponding values for RB1 $(\mathrm{P}<0.001$ for the DGS/ VCFS patients and $\mathrm{P}<0.0002$ for those with Williams syndrome; Figure 3c, d, e, f). Specifically, the frequency of SD cells for SNRPN in the samples from the DGS/VCFS patients ranged from $23 \%$ to $33 \%$ with a mean of $27.8 \pm$ $3.1 \%$, and the corresponding $R B 1$ values ranged from $29 \%$ to $45 \%$ with a mean of $35.7 \pm 5.0 \%$ (Figure $3 c$ and Figure $3 \mathrm{~d}$ ). Similarly, the frequency of SD cells in the samples from the Williams syndrome patients ranged from $12 \%$ to $31 \%$ for the SNRPN locus and from $29 \%$ to $51 \%$ for the RB1 locus with a mean of $23.4 \pm 6.5 \%$ and $36.2 \pm$ $6.7 \%$, respectively (Figure $3 e$ and Figure $3 f$ ).

The considerably low frequency of SD cells for SNRPN found in the samples of the two groups of patients with the deletion syndromes were similar to each other $(\mathrm{P}>$ $0.05)$, but each deviated significantly from those characterizing the samples of the control group $\left(\mathrm{P}<10^{-9}\right.$ and $\mathrm{P}<$ $10^{-7}$, for DGS/VCFS and Williams syndromes, respectively) (Table 1; Figure 3b, d and Figure 3f; Figure 4a). The samples of the two groups with the deletion syndromes also showed similar SD frequencies for RB1 $(\mathrm{P}>0.80)$, which were significantly higher than the corresponding $R B 1$ value observed in the control group of samples $(\mathrm{P}<$ $10^{-6}$ and $\mathrm{P}<10^{-4}$, for DGS/VCFS and Williams syndromes, respectively) (Table 1; Figure $3 \mathrm{a}, \mathrm{c}$ and Figure $3 \mathrm{e}_{\text {; Figure }}$ 4a).

When the SD frequencies for RB1 were analyzed in distinguishable (BrdU-labeled) S-phase cell populations from eight control subjects (cases C1-C8) and two patients (cases V1 and W1), it was seen that each of the two cases with the deletion syndrome revealed in the BrdU-labeled S-phase cell population a high frequency of SD cells for RB1 (42\% and 46\%, for V1 and W1, respectively), similar to the corresponding value obtained in the unlabeled Sphase cell population (Figure 5). Also, each of the control samples tested showed, for RB1, a frequency of SD cells in the S-phase labeled cell population similar to the corresponding value obtained in the unlabeled cell population (less than 24\%) (Figure 5).

As judged from the frequencies of the SS- and DD-cells for $R B 1$ in the three groups of samples tested, it appears that the increased frequency of SD cells for RB1 in the patients with a deletion syndrome results from delay in replication of a single RB1 allele. Specifically, in each group with a deletion syndrome, the frequency of SS cells for RB1 was similar to the corresponding value in the control group. However, the frequency of DD cells in each group with a deletion syndrome was significantly lower than the corresponding value in the control group (Table 1; Figure $4 \mathrm{~b}$ and Figure 4c).

When analyzing the frequencies of the SS and DD cells for $S N R P N$, it appears that the decreased frequency of SD cells observed in the DGS/VCFS patients is accompanied by a statistically significant increase in the frequency of DD 


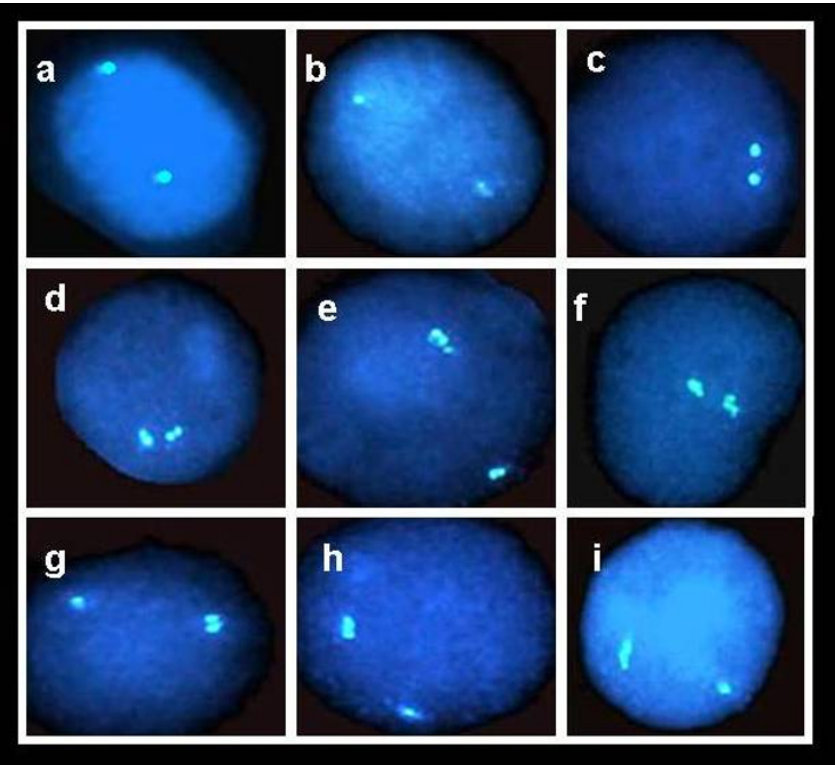

Figure 2

Fluorescent signals in PHA-stimulated lymphocytes at interphase, following FISH with RBI. (a) - (c) cells with two singlets (SS cells) in which neither allele has replicated; (d) - (f) cells with two doublets (DD cells) in which both alleles have replicated; and (g) - (i) cells with one singlet and one doublet (SD cells), which are S-phase cells in which one allele has replicated while its partner has not.

cells but not of SS cells, while the decreased frequency of SD cells observed in the Williams syndrome patients is associated with an increase in the frequency of SS cells but not of DD cells (Table 1; Figure 4b and Figure 4c).

Finally, in order to determine whether proximity to a deleted region affects the replication timing properties of an adjacent locus we studied the replication mode of the ARSA alleles in cell samples from ten DGS/VCFS patients (cases V1-V4, V11-V13 and V16-V18) and five control subjects (cases C26-C30) (Figure 1). We found that the frequency of SD cells for the ARSA locus in the samples from the patients was significantly higher than that observed in the control samples $\left(\mathrm{P}<10^{-5}\right)$ (Figure 6a and Figure 6b). It appears that the ARSA locus mimics the behavior of the RB1 locus, exhibiting SD frequencies in control samples that were significantly lower than the corresponding SNRPN values $\left(\mathrm{P}<10^{-9}\right)$, and levels in samples from subjects carrying a microdeletion that were significantly higher than the corresponding SNRPN values $(P<0.0003)$. However, the replication timing behavior of the ARSA allele located on the homologue carrying the deletion was similar to that of its counterpart situated on the normal chromosome. Therefore, it appears that the ARSA allele on the homologue with the deletion, as compared to its counterpart, showed neither a greater ten- dency for earlier replication (D signal) nor an increased preference for delayed replication (S signal). This shows that in each SD cell population of the ten DGS/VCFS cases studied, the preference for early or late replication of the ARSA allele located on the deletion-carrying homologue was random $(P>0.50$; Figure $6 c)$.

\section{Discussion}

Our results are in accordance with data showing that the $S N R P N$-imprinted locus - the hallmark of monoallelic expressed genes - normally exhibits asynchronous replication $[8,30,32]$. It has also been well documented that the expressed allele, which in the case of the SNRPN gene is the paternal allele, replicates earlier than its counterpart $[16,20]$. Clearly, the SNRPN pattern of replication differs markedly from the common synchronous pattern characterizing most genes in the human genome - namely, the biallelically expressed genes [12], exemplified here by RB1 and ARSA.

However, in the cells of microdeletion syndrome patients (of $22 \mathrm{q} 11.2$ or $7 \mathrm{q} 11.23$ ), the SNRPN gene revealed a loss of its asynchronous pattern of replication. In addition, in cells of the patients, the RB1 and the ARSA loci also exhibited replication-timing alterations, that is, both these genes demonstrated an asynchronous replication pattern. Consequently, an abnormal epigenetic profile is delineated inside genomes carrying a deleterious microdeletion, in which allelic counterparts of an imprinted locus replicate more synchronously than allelic counterparts of biallelically expressed loci.

It is clear that the considerably low frequency of SD cells for SNRPN in the deletion syndrome cases we studied cannot be attributed to a shortening of the duration of the Sphase, since there was a concomitant, significant increase in the frequency of SD cells for RB1. On the other hand, while an increase in the S-phase duration may explain the increase in the frequency of SD cells for RB1, it fails to explain the dramatic decrease in the frequency of SD observed for SNRPN. Furthermore, the S-phase-labeled cell population revealed similar values for RB1 to those obtained in the unlabeled cell population. Thereby, indicating directly that the duration of the S-phase was not associated with an alteration in the replication timing accompanying the deletion syndromes.

Since the loci studied here were selected at random, unrelated to the deletions in question, we hypothesize that other monoallelically expressed loci (in addition to $S N R P N$ ), as well as other biallelically expressed ones (in addition to RB1 and ARSA), in these genomes may also exhibit replication timing alterations. Considering the strong correlation between replication timing and epigenetic characteristics, it is reasonable to assume that the 

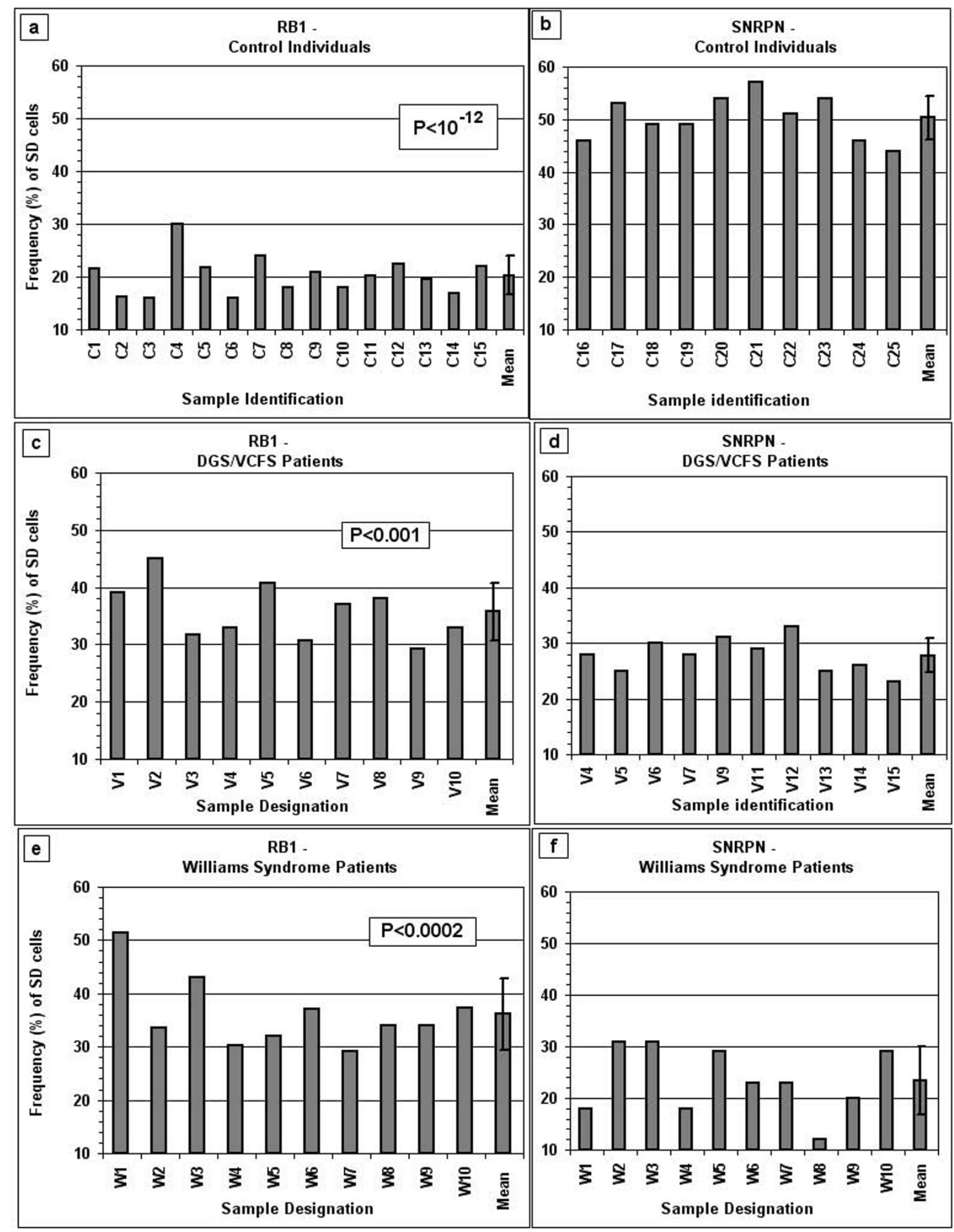

Figure 3

The frequency of SD cells for RBI and SNRPN. (a) and (b), samples from control individuals; (c) and (d), samples from patients with DGS/VCFS; and (e) and (f), samples from patients with Williams syndrome. The P values in frames (a), (c) and (e) represent the level of significance of the differences in the frequency of SD cells between RBI and SNRPN within a given group of samples. The mean frequency and standard deviation for each locus in each group of samples are also shown (last bar in each frame). 
Table I: Level of significance (P) of the differences in corresponding SD, SS and DD values between the designated groups

\begin{tabular}{|c|c|c|c|c|c|c|}
\hline & \multicolumn{2}{|c|}{ SD } & \multicolumn{2}{|c|}{ SS } & \multicolumn{2}{|c|}{ DD } \\
\hline & $R B I$ & SNRPN & $R B I$ & SNRPN & $R B I$ & SNRPN \\
\hline Control vs. DGS/VCFS & $P<10^{-6}$ & $P<10^{-9}$ & $P>0.50$ & $P>0.10$ & $P<0.05$ & $P<0.05$ \\
\hline Control vs. Williams & $P<10^{-4}$ & $P<10^{-7}$ & $P>0.05$ & $P<0.0005$ & $P<10-4$ & $P>0.40$ \\
\hline DGS/VCFS vs. Williams & $P>0.80$ & $P>0.05$ & $P>0.40$ & $P<0.01$ & $P>0.05$ & $P<0.05$ \\
\hline
\end{tabular}

Control group (cases $\mathrm{CI}-\mathrm{CI} 5$ for RBI and CI6-C25 for SNRPN); DGS/VCFS group (cases VI-VI0 for RBI, and cases VI, V5-V7, V9, VII-VI5 for SNRPN); and Williams syndrome group (cases WI-WI0).

global replication timing modifications displayed within the deletion-carrying genomes lead to major alterations in the epigenomic features. This reasoning is in line with the view that the replication timing is both the cause and the consequence of the chromatin structure of a gene [9]. We have shown here that alleles of a gene that is normally subject to monoallelic expression fail to keep the inherent functional asymmetry between the allelic counterparts necessary for establishing normal phenotypes. Conversely, the normally biallelically expressed loci fail to keep the functional symmetry between parental counterparts required to fulfill Mendelian laws. This loss of symmetry in deletion-carrying genomes for alleles of biallelically expressed genes co-occurring with loss of the inherent capability of exclusively specialized genes for allele-specific behaviour, may explain the numerous and variable, non-deletion-specific abnormal traits that accompany both of these microdeletion syndromes.

Interestingly, in the DGS/VCFS group the loss of replication asynchrony observed for the SNRPN locus is mainly due to an advanced replication of the normally late-replicating (maternal) allele, while the loss of asynchrony for SNRPN in the Williams syndrome patients is mostly associated with a delay in the replication timing of the normally early-replicating (paternal) SNRPN allele. Yet, it is illogical to assume that the deletion in question "recognizes" whether a far-removed allele is maternal or paternal in origin through some parent-of-origin inborn signal. The differentiation is possibly based on the epigenetic dif-

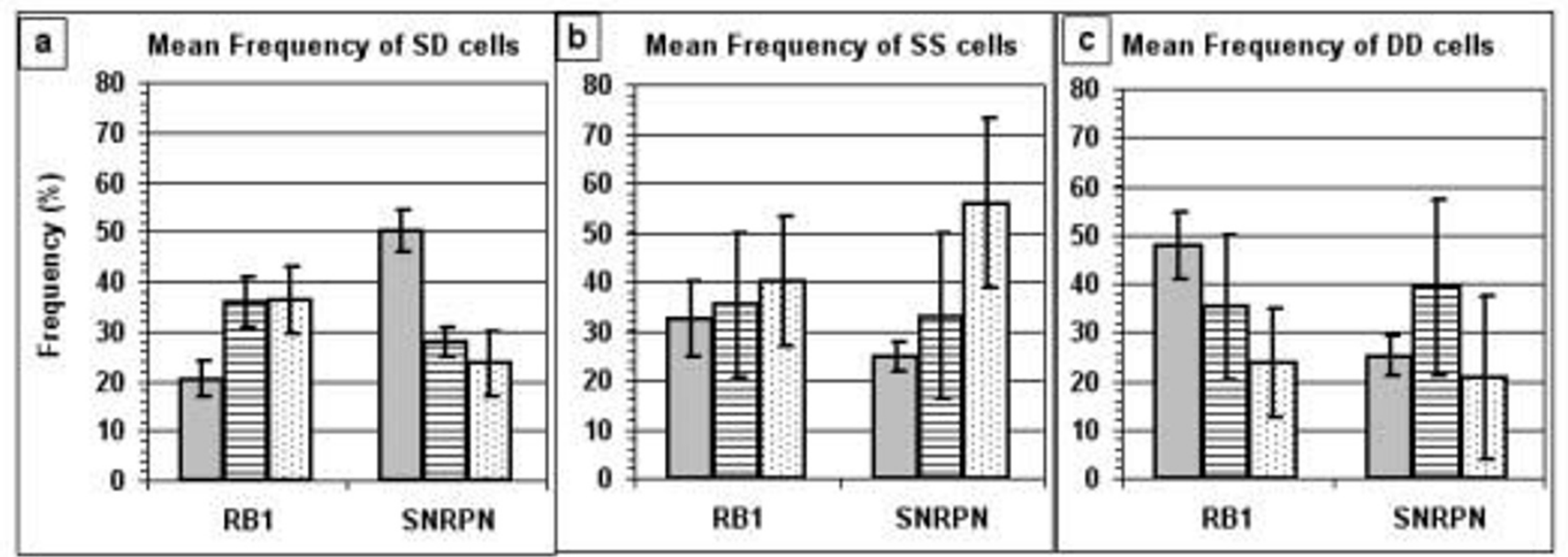

\section{Control घDGSNCFS घWilliams}

\section{Figure 4}

Mean frequency values for SD, SS and DD cells for RBI and SNRPN in control and patient samples. Solid bars present the control sample (cases $\mathrm{CI}-\mathrm{CI} 5$ for $R B I$ and $\mathrm{Cl}$-C25 for SNRPN); striped bars present the sample of patients with DGS/VCFS (cases VI-VIO for RBI, and VI, V5-V7, V9 and VII-VI5 for SNRPN); and dotted bars present the sample of patients with Williams syndrome (cases WI-WI0). 


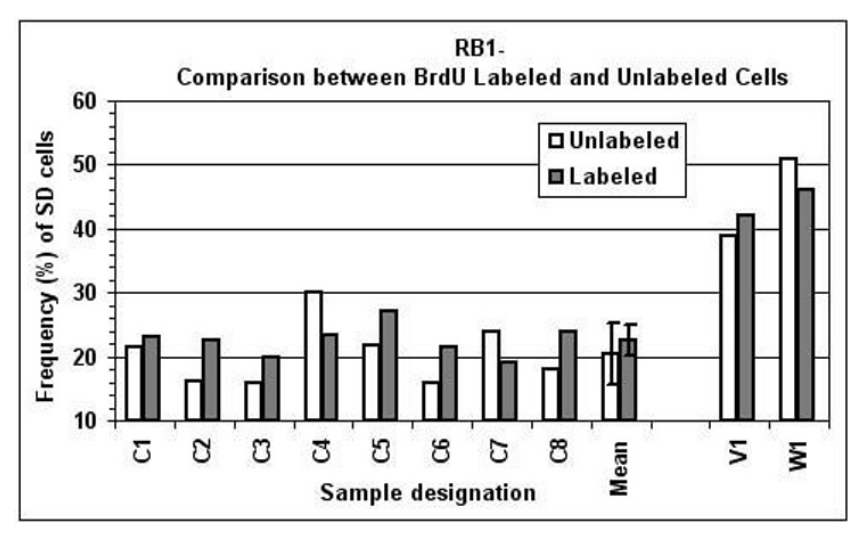

Figure 5

Frequency of SD cells for RBI in BrdU-labeled and unlabeled cell populations. Control individuals (cases $\mathrm{CI}-\mathrm{C} 8$ ), a patient with DGS/VCFS (case VI) and a patient with Williams syndrome (case WI). Each sample is shown with two SD values for $R B I$, one in an unlabeled cell population (open bars) and the second in a BrdU labeled population (solid bars).

ferences characterizing alleles of the imprinted locus that were established in the parental germ cells prior to fertilization [35]. Whatever the reason for the differences in $S N R P N$ replication behavior in the two deletion syndromes, it clarifies how loss of asynchronous replication, even for a single and specific monoallelic gene, may lead (in addition to non-deletion-specific malformations) to syndrome-specific abnormal phenotypic expression. It is interesting to note that relaxation of imprinting, arising either from activation of the silent allele or from silencing of the normally active allele was documented previously in association with the numerous, variable epigenetic alterations characterizing malignant phenotypes [36].

One could speculate, therefore, that each microdeletion shows specificity toward a particular epigenetic profile, and if the two parental allelic counterparts retain the same structure, they are not differentiated specifically by the deletion in question. This view is in accordance with the replication pattern of the ARSA locus, which replicates synchronously in normal cells, and as such its alleles are expected to have initially (at fertilization) similar epigenetic structures. Indeed, our results show that in cells carrying the 22q11.2 deletion, the asynchronous replication of the ARSA locus resulted from a random preference of an allele for early or late replication, probably determined de-novo for each replication cycle. This is inferred from the observation that in half (about 50\%) of each population of SD cells from patients with the 22q11.2 deletion, the allele located on the homologue carrying the deletion was the early-replicating allele, while in the other half its counterpart was the early one. Additionally, it indicates that an allele located distally to the deleted region, on the same chromosomal arm, behaves similarly to its counterpart located on the normal arm. Together, our results disagree with the notion that asynchronous replication in a deletion-carrying genome results merely from a type of position-effect (change in position along the chromosome) of the allele located on the deletion-carrying homologue [37]. Our results are in agreement with a short report claiming that the presence of a microdeletion within the genome increases the level of replication asynchrony of biallelically expressed genes not located on the deletioncarrying chromosome [38]. Furthermore, we show here that the asynchronous pattern of replication of biallelically expressed loci, exemplified by $R B 1$ in cells with a 22q11.2 deletion, as well as those with a 7q11.23 deletion, appears to result from a delay in replication of a single allele (inferred by a decrease in the frequency of DD cells but not of SS cells). Yet, based on the replication pattern of the ARSA locus, which mimics the replication behavior of the RB1 locus, it is reasonable to assume that in a deletion-carrying genome, the preference of a specific allele of a biallelically expressed gene for delayed replication is also random. It is therefore independent of any kind of position effect. This is supported by a study in cells with a 22q11.2 deletion that the TUPLE1 allele, situated on the intact chromosome 22 within the 22 q11.2 region, presents a delayed replication timing [6].

Asynchronous replication of biallelically expressed loci has been reported in cells from patients carrying an aneuploidic loss of the whole X-chromosome (Turner syndrome, shown in peripheral blood cells) [39] or the gain of an autosome (trisomies 21, 18 and 13, shown in amniotic fluid cells) $[40,41]$. In these whole-chromosome aneuploidies [39-41], the biallelically expressed loci that were shown to change their characteristic synchronous pattern of replication to an asynchronous one were not associated with the lost or gained chromosome. Taken together, it is reasonable to assume that the phenomenon of replication-timing alteration in loci unrelated to the imbalancecausing-DNA sequence is not specific to the size or type of the lost/gained chromosomal segment (or whole chromosome). Thus, the view that a specific gene(s) assigned to the missing segment, in each of the microdeletion genotypes studied here, is the sole cause of the global replication timing aberrations is weakened. This reasoning remains coherent even in light of the information that the deletion of the DGS/VCFS is gene-dense [reviewed in [6]] and the deletion of the Williams syndrome carries a gene (RFC2) that codes for a subunit of a replication factor, a part of the multimeric complex involved in DNA elongation during replication [7]. Furthermore, even the view that replication-timing alterations are associated with an existing predisposition factor that destabilizes the integrity of the genome appears to be incompatible with the 


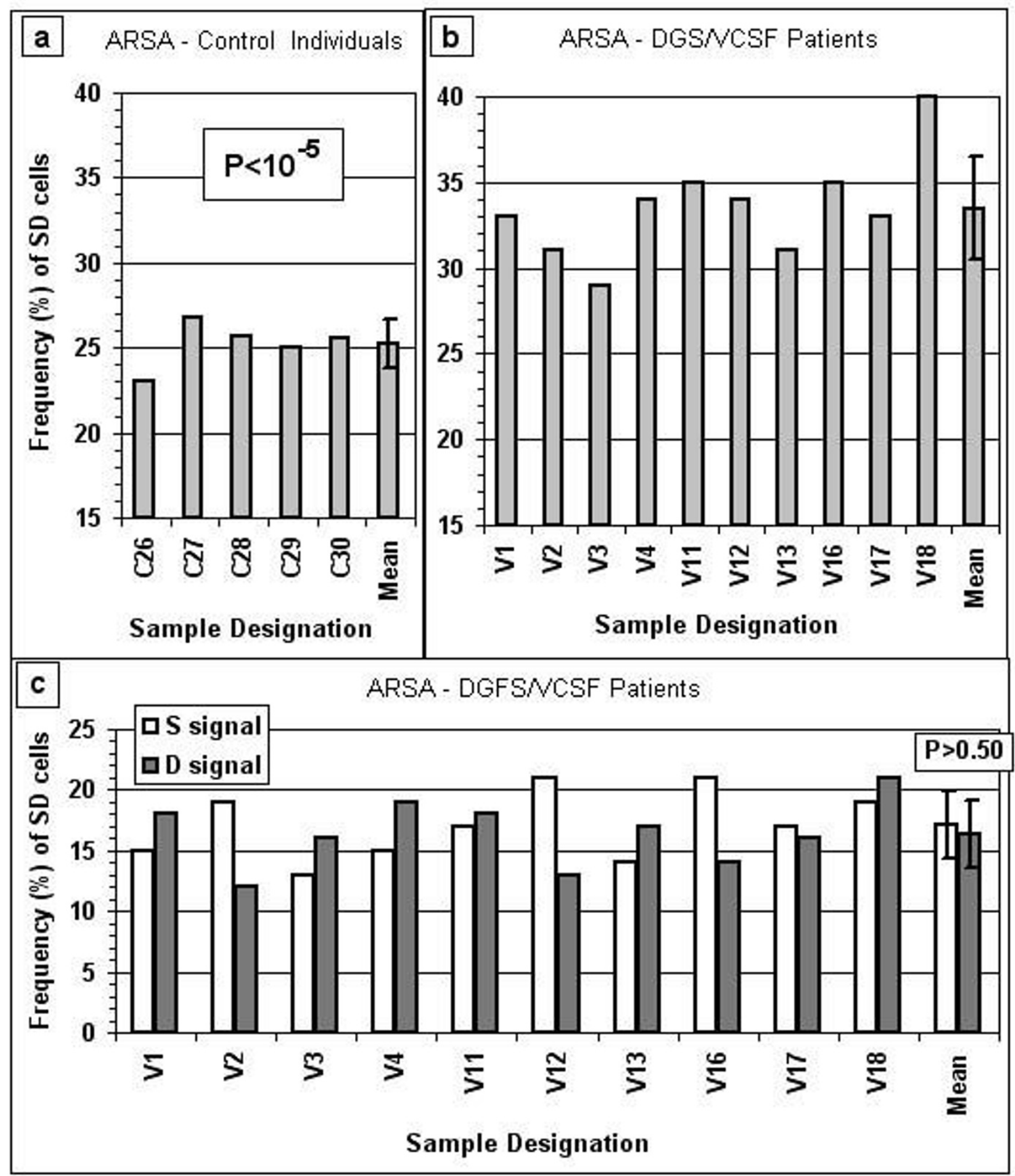

Figure 6

Frequency of SD cells for ARSA in samples from control individuals and from patients with DGS/VCFS. In frame (c) the frequency of SD cells in each individual sample was separated into two sub-categories: cells in which the ARSA signal of the deletion-carrying homologue shows a singlet (S-signal, open bar) and those in which the ARSA signal of the deletion-carrying chromosome reveals a doublet (D-signal, solid bar). The mean frequencies and standard deviations for each group of samples are also given (last bar(s) in each frame). The $P$ value in frame (a) represents the significance of the difference between the values in frames (a) and (b); the $P$ value in frame (c) shows the significance of the difference between the $S$ and $D$ values for the $A R S A$ signal of the deletion-carrying homologue within the total ARSA SD cell population. 
current data; any potential destabilizing mechanism leading to a segmental deletion/duplication $[4,5,42,43]$ is entirely different from that giving rise to the loss/gain of a whole chromosome [44]. Therefore, it is more logical to assume that the global alteration in allelic replication timing results from the aneuploidic status itself. This is in accordance with data showing that peripheral blood cells derived from individuals with normal karyotypes, which show increased levels of sporadic (non-chromosome specific) aneuploidy due to malignancy, exhibit replication timing modification of various loci unrelated to the missing/gained chromosomes [45-47]. This further strengthens the notion that the nature of the missing/gained genes assigned to the lost/gained DNA is not imperative to the replication anomaly described here.

Yet, the non-locus-specific aberrant replication timing in the cells of cancer patients was reverted to normal in the presence of 5-azacytidine, a classical methylation-blocking agent, recently approved as an anti-cancer drug [48], thereby linking replication timing alteration to methylation capacity $[46,47,49]$. This agrees with our previous studies in cells of mutation carriers [25] and patients with the fragile $\times$ syndrome [50], where we showed that the mutated (FMR1) allele (characterized by highly methylated tri-nucleotide repeats) demonstrated delayed replication, which was restored by the application of a demethylating drug [50]. Similarly, replication studies in cells of girls with Rett syndrome link loss of asynchronous replication of X-linked genes with DNA methylation [51]. Rett syndrome is caused by a mutation in an X-linked gene (MECP2) that normally codes for a protein capable of binding to methylated DNA; it therefore takes part in the line of events leading to transcriptional silencing of $\mathrm{X}$ inactivated genes [reviewed in [52]].

The involvement of methylation capacity with replication timing is not surprising since allelic counterparts, which exhibit normally asynchronous replications are most often differentially methylated $[8,12]$. It is therefore reasonable to assume that also the aberrant replication accompanying a microdeletion is associated with aberrant methylation. It is yet not clear if independent mechanisms control allelic replication timing (synchronous or asynchronous) and methylation capacity (similar or different). Nevertheless, the non-random spatial organization of homologous DNA segments in the diploid nucleus was suggested to play a critical role in both replication timing and methylation capacity $[8,12]$. Furthermore, numerous studies also show that DNA spatial organization in the diploid nucleus is most crucial for epigenomic stability and proper gene functioning [reviewed in [53]]. Therefore, it is tempting to speculate that the loss/gain of a single chromosomal segment (or whole chromosome) disrupts the structural unity of the diploid status, altering the overall spatial organization of chromosomes within the interphase nucleus. This in turn leads to (non-gene specific) epigenetic alterations of genes situated on chromosomes other than those carrying the aberration, as well as those located on the aberrant chromosome. This speculation is supported in part by data showing that in cells of DGS/VCFS patients the intact chromosome 22 displays a specific (peripheral) nuclear location, which is different from that observed in normal cells, for two intact (fully homologous) chromosomes 22 [6].

Whatever the mechanism underlining abnormal allelic replication timing, the epigenetic alteration delineated by the deletion is not deletion-specific, and probably accompanies any loss/gain of a whole chromosome or of a small chromosomal segment. As such, abnormal allelic replication timing offers a potential epigenetic marker, a kind of a preliminary hint to suspect the existence of any type of chromosomal imbalance. For instance, the replicationtiming test can be used to investigate unexplained cases of mental retardation as well as other phenotypic abnormalities suspected to arise from minute segmental aneuploidy that cannot be seen by conventional karyotyping $[1,54]$. Specifically, cases showing a higher level of asynchrony for a biallelic gene compared to that observed (in the very same cell sample) for a monoallelic one, call for further investigations. Moreover, the global epigenetic alteration, as described here in PHA-stimulated lymphocytes of microdeletion-carrying patients, probably appears in prenatal tissues as well, as can be inferred from the high asynchronous replication of biallelically expressed loci observed previously in the amniotic fluid cells of cases with trisomies 21, 18 and $13[40,41]$. It is therefore reasonable to assume that observation of a reversal of the replication patterns in amniotic cells might mark a fetus that has inherited a chromosomal imbalanced genome and thus, it calls for further attention. One could seek to apply more laborious and costly DNA analyses for examining the integrity of the entire chromosomal complement. These might include high-resolution array-based comparative genomic hybridization techniques $[2,3,54]$, capable of characterizing the specific DNA segment incriminated in a chromosomal imbalance.

Finally, we would like to refer shortly to the abundance of submicroscopic copy number variations (CNV) of DNA segments ranging from a few kilobases $(\mathrm{kb})$ to megabases $(\mathrm{Mb})$ in size recently discovered in the human genome, whose impact upon the genome is still undefined $[55,56]$. It is worth examining whether those variations are accompanied by replication-timing alterations and whether such alterations could be of use in differentiating between deleterious and harmless CNVs. 


\section{Conclusion}

Within genomes carrying a deleterious microdeletion, an abnormal epigenetic profile is delineated in which allelic counterparts of an imprinted gene, not directly associated with the missing DNA, replicate more synchronously than allelic counterparts of biallelically expressed loci. This epigenetic aberration, which appears to be non-deletion specific, is in line with the numerous non-deletion specific phenotypic abnormalities usually accompanying each microdeletion syndrome. Such an aberration, which is easily detected by cytogenetic means, offers a potential preliminary screening tool for such conditions, in effect, an "epigenotype-first approach" that can be performed without any prior information as to the specific genetic nature of the underlying abnormality.

\section{Competing interests}

The authors declare that they have no competing interests.

\section{Authors' contributions}

JY and IA have contributed equally to this work. Both, participated in the design of the study, carried out most of the cytogenetic work, performed the statistical analyses and helped to draft the manuscript. AR and JF carried out some of the cytogenetic studies and participated in the collection of samples. MS was involved in the design of the study. LA conceived of the study, participated in its design, and coordinated and drafted the manuscript. All authors read and approved the final manuscript.

\section{Acknowledgements}

This work fulfils in part the research requirements for the MSc degree of IA. This research was funded in part by the Yeshaya Horovitz Grant for Neurogenetics Research.

\section{References}

I. Shaffer LG, Ledbetter DH, Lupski JR: Molecular cytogenetics of contiguous gene syndromes: mechanisms and consequences of gene dosage imbalance. In Metabolic and Molecular Basis of Inherited Disease Edited by: Scriver CR, Beaudet AL, Sly WS, Valle D, Childs B, Kinzler KW, Vogelstein B. New York: McGraw Hill; 200I:I29|-1324.

2. Bejjani BA, Saleki R, Ballif BC, Rorem EA, Sundin K, Theisen A, Kashork CD, Shaffer LG: Use of targeted array-based CGH for the clinical diagnosis of chromosomal imbalance: is less more? Am J Med Genet 2005, I34A:259-267.

3. Zahir F, Friedman JM: The impact of array genomic hybridization on mental retardation research: a review of current technologies and their clinical utility. Clin Genet 2007, 72:27I-287.

4. Baumer A, Dutly F, Balmer D, Riegel M, Tukel T, Krajewska-Walasek $M$, Shinzel AA: High level of meiotic crossovers at the origin of the 22qII.2 and 7qII.23 deletions. Hum Mol Genet 1998, 7:887-894.

5. Edelmann L, Pandita RK, Spiteri E, Funke B, Goldberg R, Palanisami N, Chaganti RSK, Magenis E, Shprintzen RJ, Morrow BE: A common molecular basis for rearrangement disorders on chromosome 22q I I. Hum Mol Genet 1999, 8: I I57-I I67.

6. D'Antoni S, Mattina T, Di Mare P, Federico C, Motta S, Saccone S: Altered replication timing of the HIRA/Tuplel locus in the DiGeorge and Velocardiofacial syndromes. Gene 2004, 333: $111-119$.
7. Peoples R, Perez-Jurado L, Wang Y-K, Kaplan P, Francke U: The gene for replication factor $C$ subunit 2 (RFC2) is within the 7 I I.23 Williams syndrome deletion. Am J Hum Genet 1996, 58: |370- I373.

8. Goldmit M, Bergman Y: Monoallelic gene expression: a repertoire of recurrent themes. Immunol Rev 2004, 200:197-2I4.

9. Gilbert DM: Replication timing and transcriptional control: beyond cause and effect. Curr Opin Cell Biol 2002, 14:377-383.

10. McNairn AJ, Gilbert DM: Epigenomic replication: linking epigenetics to DNA replication. BioEssays 2003, 25:647-656.

II. Woodfine K, Fiegler H, Beare DM, Collins JE, McCann OT, Young BD, Debernardi S, Mott R, Dunham I, Carter NP: Replication timing of the human genome. Hum Mol Genet 2004, 1 3: 1 91-202.

12. Goren E, Cedar H: Replication by the clock. Nat Rev Mol Cell Biol 2003, 4:25-32.

13. Morishima A, Grumbach MM, Taylor JH: Asynchronous duplication of human chromosomes and the origin of sex chromatin. Proc Natl Acad Sci USA 1962, 48:756-763.

14. Priest JH, Heady JE, Priest RE: Delayed onset of replication of human X chromosomes. J Cell Biol 1967, 35:483-487.

15. Selig S, Okumura K, Ward DC, Cedar H: Delineation of DNA replication time zones by fluorescence in situ hybridization. EMBO J 1992, II:1217-1225.

16. Kitsberg D, Selig S, Brandeis M, Simon I, Keshet I, Driscoll DJ, Nicholls $\mathrm{RD}$, Cedar H: Allele-specific replication timing of imprinted gene regions. Nature 1993, 364:459-463.

17. Boggs BA, Chinault AC: Analysis of DNA replication by fluorescence in situ hybridization. Methods 1997, 13(3):259-270.

18. Simon I, Tenzen T, Reubinoff BE, Hillman D, McCarrey JR, Cedar H: Asynchronous replication of imprinted genes is established in the gametes and maintained during development. Nature 1999, 40 I:929-932

19. Knoll JHM, Cheng S-D, Lalande M: Allele specificity of DNA replication timing in the Angelman/Prader-Willi syndrome imprinted chromosomal region. Nat Genet 1994, 6:41-46.

20. Gunaratne PH, Nakao M, Ledbetter DH, Sutcliffe JS, Chinault AC: Tissue-specific and allele-specific replication timing control in the imprinted human Prader-Willi syndrome region. Genes Dev 1995, 9:808-820.

21. LaSalle JM, Lalande M: Domain organization of allele-specific replication within the GABRB3 gene cluster requires a biparental I5q I I-I 3 contribution. Nat Genet 1995, 9:386-394.

22. White LM, Rogan PK, Nicholls RD, Wu BL, Korf B, Knoll JHM: Allele specific replication of 15 q I I-q 13 loci: a diagnostic test for uniparental disomy. Am J Hum Genet 1996, 59:423-430.

23. Torchia BS, Call LM, Migeon BR: DNA replication analysis of FMRI, XIST, and factor $8 \mathrm{C}$ loci by FISH shows nontranscribed X-linked genes replicate late. Am J Hum Genet 1994, 55:96-104.

24. Boggs BA, Chinault AC: Analysis of the replication timing properties of human $X$-chromosomal loci by fluorescence in situ hybridization. Proc Natl Acad Sci USA 1994, 91:6083-6087.

25. Yeshaya J, Shalgi R, Shohat M, Avivi L: FISH-detected delay in replication timing of mutated FMRI alleles on both active and inactive X-chromosomes. Hum Genet 1999, 105:86-97.

26. Chess A, Simon I, Cedar H, Axel R: Allelic inactivation regulates olfactory receptor gene expression. Cell 1994, 78:823-834.

27. Hollander GA, Zuklys S, Morel C, Mizoguchi E, Mobisson K, Simpson S, Terhorst C, Wishart W, Golan DE, Bhan AK, Burakoff SJ: Monoallelic expression of the interleukin-2 locus. Science 1998, 279:21|8-2121.

28. Mostoslavsky R, Singh N, Tenzen T, Goldmit M, Gabay C, Elizur S, Qi $P$, Reubinoff BE, Chess A, Cedar H, Bergman Y: Asynchronous replication and allelic exclusion in the immune system. Nature 200I, 4I 4:22I-225.

29. Simom I, Tenzen T, Mostosslavsky R, Fibach E, Lande L, Milot E, Gribnau J, Grosveld F, Fraser P, Cedar H: Developmental regulation of DNA replication timing at the human globin locus. $E M B O$ J 200I, 20:6150-6157.

30. Ensminger AW, Chess A: Coordinated replication timing of monoallelically expressed genes along human autosomes. Hum Mol Genet 2004, I 3:65 I-658.

31. Goldmit M, Ji Y, Skok J, Roldan E, Jung S, Cedar H, Bergman Y: Epigenetic ontogeny of the Igk locus during $B$ cell development. Nat Immunol 2005, 6: 1 98-203. 
32. Gribnau J, Hochedlinger K, Hata K, Li E, Jaenisch R: Asynchronous replication timing of imprinted loci is independent of DNA methylation, but consistent with differential subnuclear localization. Genes Dev 2003, 17:759-773.

33. Bergman $\mathrm{Y}$, Cedar $\mathrm{H}$ : A stepwise epigenetic process controls immunoglobulin allelic exclusion. Nat Rev Immunol 2004, 4:754-76I.

34. Gimelbrant AA, Ensminger AW, Qi P, Zucker J, Chess A: Monoallelic expression and asynchronous replication of p I 20 Catenin in mouse and human cells. J Biol Chem 2005, 280:1354-1359.

35. Morgan HD, Santos F, Green K, Dean W, Reik W: Epigenetic reprogramming in mammals. Hum Mol Genet 2005, I 4:R47-R58.

36. Feinberg AP, Tycko B: The history of cancer epigenetics. Nat Rev Cancer 2004, 4: I43-I 53

37. Ofir R, Wong ACC, Mcdermid HE, Skorecki KL, Selig S: Position effect of human telomeric repeats on replication timing. Proc Natl Acad Sci USA 1999, 96: I |434-I I 439.

38. Amiel A, Reish O, Gaber E, Masterman R, Tohamy T, Fejgin MD: Asynchronous replication of alleles in genomes carrying a microdeletion. Isr Med Assoc J 2002, 4:702-705.

39. Reish O, Gal R, Gaber E, Sher C, Bistritzer T, Amiel A: Asynchronous replication of biallelically expressed loci: a new phenomenon in Turner syndrome. Genet Med 2002, 4:439-443.

40. Amiel A, Avivi L, Gaber E, Fejgin MD: Asynchronous replication of allelic loci in Down syndrome. Eur J Hum Genet 1998 , 6:359-364

4I. Amiel A, Korenstein A, Gaber E, Avivi L: Asynchronous replication of alleles in genomes carrying an extra autosome. Eur J Hum Genet 1999, 7:223-230.

42. Shaw C], Lupski JR: Implication of human genome architecture for rearrangement-based disorders: the genomic basis of disease. Hum Mol Genet 2004, I3:R57-R64.

43. Baumer A, Rigel M, Schinzel A: Non-random asynchronous replication at $22 \mathrm{qI} I .2$ favors unequal meiotic crossover leading to the human 22q I I.2 deletion. J Med Genet 2004, 4I:4I3-420.

44. Hassold T, Hunt P: To Err (meiotically) is human: the genesis of human aneuploidy. Nat Rev Genet 200I, 2:280-29I.

45. Litmanovitch T, Altaras MM, Dotan A, Avivi L: Asynchronous replication of homologous -satellite DNA loci in man is associated with non-disjunction. Cytogenet Cell Genet 1998, 81:26-35.

46. Korenstein-llan A, Amiel A, Lalezari S, Lishner M, Avivi L: Allele specific replication associated with aneuploidy in blood cells of patients with hematological malignancies. Cancer Genet Cytogenet 2002, 139:97-103.

47. Dotan ZA, Dotan A, Ramon J, Avivi L: Altered mode of allelic replication accompanied by aneuploidy in peripheral blood lymphocytes of prostate cancer patients. Int J Cancer 2004, I I I:60-66.

48. Garcia-Manero G: Demethylating agents in myeloid malinancies. Curr Opin Oncol 2008, 20:705-7I0.

49. Dotan ZA, Dotan A, Ramon J, Avivi L: Aberrant allele-specific replication, independent of parental origin, in blood cells of cancer patients. BMC Cancer 2008, 8:390. doi:I0.I I86/I47I-24078-390

50. Yeshaya J, Shalgi R, Shoha tM, Avivi L: Replication timing of the various FMRI alleles detected by FISH: inferences regarding their transcriptional status. Hum Genet 1998, 102:6-14.

5I. Vorsanova SG, Yurov YB, Kolotii AD, Soloviev IV: FISH analysis of replication and transcription of chromosome $X$ loci: new approach for genetic analysis of Rett syndrome. Brain \& Development 200I, 23:SI9I-SI95.

52. Caballero IM, Hendrich $B$ : MeCP2 in neurons: closing in on the causes of Rett syndrome. Hum Mol Genet 2005, I 4:R I 9-R26.

53. Prada L, Misteli T: Chromosmoe positioning in the interphase nucleus. Trends Cell Biol 2002, I 2:425-432.

54. Shaffer LG, Theisen A, Bejiani BA, Ballif BC, Aylsworth AS, Lim C, McDonald M, Ellison JW, Kostiner D, Saitta S, Shaikh T: The discovery of microdeletion syndromes in the post-genomic era: review of the methodology and characterization of a new I q4 I q42 microdeletion syndrome. Genet Med 2007, 9:607-6I6.

55. Feuk $L$, Carson AR, Scherer SW: Structural variation in the human genome. Nat Rev Genet 2006, 7:85-97.

56. Hurles ME, Dermitzakis ET, Tyler-Smith C: The functional impact of structural variation in humans. Trends Genet 2008, 24:238-244.
Publish with Biomed Central and every scientist can read your work free of charge

"BioMed Central will be the most significant development for disseminating the results of biomedical research in our lifetime. "

Sir Paul Nurse, Cancer Research UK

Your research papers will be:

- available free of charge to the entire biomedical community

- peer reviewed and published immediately upon acceptance

- cited in PubMed and archived on PubMed Central

- yours - you keep the copyright 